\title{
Observational Data Collection of Environmental and Behavioral Characteristics: Strengths, Limitations, and Implications for Health Communication
}

\author{
Sonali Rajan', Corey H Basch ${ }^{2 *}$ and Danna Ethan ${ }^{3}$
}

${ }^{1}$ Department of Health and Behavior Studies, Teachers College, Columbia University, New York, New York, USA

${ }^{2}$ Department of Public Health, William Paterson University, Wayne, New Jersey, USA

${ }^{3}$ Department of Health Sciences, Lehman College, The City University of New York, Bronx, New York, USA

\section{Introduction}

The collection of observational data is crucial to understanding both the environmental and behavioral characteristics of individuals and communities. The implications of the observational data collection process, particularly in contributing to current health communication research and public health outreach efforts, is significant. Specifically, the use of such methods has become increasingly important as disease and injury prevention efforts are grounded in our understanding of human behavior and the environmental factors that affect and shape them [1]. Further, research has well documented the limitations of other forms of data collection, for example survey methods [2] or retrospective analyses [3], that observational data collection can help supplement. Perhaps most importantly, this increased understanding guides both the content and medium public health practitioners and educators utilize to most effectively communicate to at- risk populations [4].

Observational data collection is typically classified into two distinct categories: direct versus indirect [5]. In the former, researchers observe specific behaviors or processes and the latter allows researchers to observe the outcome of a set of specific behaviors [5]. It should be noted that observed interactions also include the relationship between individuals and their environment [5]. We expand upon this definition to further include the observation of specific environmental characteristics and the observation of products. It should also be acknowledged that the collection and analyses of qualitative data are extremely important to providing context and nuance regarding particular individuals and communities. For the purposes of this commentary, however, our focus is on the collection of quantitative data via observational methods and the translation of such data into findings that can be used to inform broader public health programmatic and policy efforts.

This commentary offers specific examples of environmental and behavioral observational data collection processes that the study's authors have recently implemented. We also describe the strengths and limitations associated with this methodology. Implications for mass communication in the context of public health efforts are discussed.

\section{Observational Data Collection Process}

\section{Environmental characteristics}

The identification of the built environment as a critical factor that directly affects and shapes human behavior has been well established in the literature and in the context of a range of health issues. These characteristics are addressed most frequently when discussing the obesity epidemic and related co-morbidities [6] and patterns of violence in specific communities [7]. The definition of the built environment, which originally focused on specific factors (e.g. availability of sidewalks to increase physical activity [8] and green space in urban environments to improve the quality of neighborhoods [9], has since expanded to also include factors such as the presence of vending machines in schools [10] and media marketing strategies [11].

The authors recently conducted three separate studies (two publications cited, one abstract currently under review) using observational data collection methods to accomplish the following: 1) describe marketing strategies utilized among potentially toxic, nonfood products [12] 2) describe product characteristics [12], 3) identify the placement of promotions in fast-food settings that increase the consumption of processed meals [13], and 4) describe the availability of nutrition information to improve consumer choices when in a fast-food environment [13]. Together the collection of these data have allowed for a more nuanced understanding of environmental characteristics that shape and inform quality of health. In the former studies, implications of product marketing strategies specifically tailored to children were identified and described via a comprehensive and exhaustive coding process. In the latter study, observational data collected from 70 fast food restaurants in New York City (NYC) furthered a discussion regarding the environmental characteristics that affect consumer food choices. In all instances, these observational data contributed to gaps in health communication knowledge; specifically, addressing what kinds communication efforts are needed and where to counter environmental characteristics that contribute to poor quality of health.

\section{Behavioral characteristics}

More recently, the study's authors collected data on technologyrelated distracted walking behaviors (manuscript under review) and bike helmet use among pedestrians in NYC (manuscript in progress). In the former study, the researchers observed over 3,500 pedestrians using dangerous crosswalks in Manhattan, NYC. Pedestrians' use of headphones and either talking on or looking down at a mobile phone or smartphone were coded by the authors. To ensure reliability given the large numbers of pedestrians crossing the street during light changes, one author coded the total number of pedestrians crossing at each light change while the second author coded for distracted behaviors. A similar methodology was utilized when collecting pilot data on bike

*Corresponding author: Corey $\mathrm{H}$ Basch, Assistant Professor, Department of Public Health, William Paterson University, Wayne, New Jersey, USA, Tel: 973720-2603; E-mail: BASCHC@wpunj.edu

Received September 10, 2013; Accepted September 11, 2013; Published September 12, 2013

Citation: Rajan S, Basch CH, Ethan D (2013) Observational Data Collection of Environmental and Behavioral Characteristics: Strengths, Limitations, and Implications for Health Communication. J Mass Communicat Journalism 3: e141. doi:10.4172/2165-7912.1000e141

Copyright: ( 2013 Rajan S, et al. This is an open-access article distributed under the terms of the Creative Commons Attribution License, which permits unrestricted use, distribution, and reproduction in any medium, provided the original author and source are credited. 
Citation: Rajan S, Basch CH, Ethan D (2013) Observational Data Collection of Environmental and Behavioral Characteristics: Strengths, Limitations, and Implications for Health Communication. J Mass Communicat Journalism 3: e141. doi:10.4172/2165-7912.1000e141

helmet use: trained coders observed the prevalence of bike helmet use among a representative sample of participants participating in NYC's CitiBike program. Most recently, the authors have initiated a new study, whereby they have added videotaping to increase coding capabilities when observing distracted cycling behaviors (e.g. use of headphones, helmet use) among bikers in NYC (manuscript in progress).

\section{Strengths}

The results of the aforementioned research confirm a number of strengths associated with observational data collection. First, and perhaps most importantly, this process allows researchers to observe environmental characteristics (product details, marketing strategies, information availability) and behavioral characteristics in their natural environment. Data can be collected as events unfold versus relying on reports of what has already happened; or, in the instance of survey methodology, relying primarily on self-reported information. Prevalence of distracted walking or cycling behaviors, for example, were documented as they occurred. Similarly, product characteristics and marketing strategies were documented in real time, which also allowed for increased accuracy and relevancy of the data. Second, observations are not limited solely to behaviors and the environment. Additional factors and possible covariates at both the individual and environmental level can be coded, including gender, location, socioeconomic status, and even body mass index [14]. Observational data collection also provides opportunities to document the context of specific behaviors. For example, when documenting nutrition information availability in an urban fast food environment, the researchers were also able to document variations in pricing at each restaurant [13]. Lastly, the expanding use of technology in research (e.g. videotaping) ensures that large amounts of data may be collected at any given point. Together these efforts will ultimately contribute to improving health communication efforts; particularly those targeting specific segments of the population.

\section{Limitations}

There are also limitations of observational research to consider. First, individuals who are or become aware of being observed may change their behavior as a result of being observed. While that did not appear to significantly affect the quality of data collected in any of the aforementioned studies, this could become an issue if observational data were to be collected in a more intimate setting (e.g. when establishing the prevalence of individuals washing their hands after using the bathroom [15]. Second, attention must be paid by researchers to the evolving nature of marketing strategies, products, and information availability. For instance, a promotion for fruit smoothies or iced coffee drinks may only be offered during the summer months. Therefore, the need to collect data within a relatively short time frame is crucial. Third, because the surrounding environment is not controlled during the process of observational data collection, it can be difficult to ensure representativeness of the data. It is necessary to therefore obtain a large enough sample size that can then accurately be generalized to the broader population. Lastly, the process of collecting observational data, which relies almost exclusively on coding efforts, is subject to a great deal of variance, particularly when multiple details are being recorded. Therefore, there is a need for multiple coders to ensure reliability of the data.

\section{Discussion}

Mass communication efforts are a crucial component of public health information sharing and dissemination [16]. Such efforts have been utilized to promote information among communities about vaccines [17], emergency preparedness [18], and HIV prevention [19]. The need to understand behavioral and environmental contextual nuances of specific communities and health issues is necessary if such efforts are to be truly successful. Observational data can help inform the development of public service announcements, social networking efforts, questions to be used during focus groups or other types of qualitative interviews, and online or print-based media campaigns.

Observational data collection, when implemented thoughtfully and with rigor, can contribute significantly to current health communication research and public health outreach efforts. As described above, the authors recently published two studies $[12,13]$, have two studies under review, and are in the process of implementing two more studies that have each successfully utilized observational data collection methods to document environmental and behavioral characteristics. Though some limitations exist with this process, there are many strengths of observational data collection; most notably, data are collected in real time and in one's natural environment, and contextual factors can be included in the final analysis and interpretation of the data. These strengths therefore ought to be considered by researchers looking to enhance current study designs and/or inform health communication initiatives.

\section{References}

1. Johns Hopkins Center for Injury Research and Policy (2013) NYC Department of Health and Mental Hygiene, Society for Public Health Education, Active Design Supplement.

2. Zonfrillo M, Wiebe D (2011) Survey science in pediatric emergency medicine Pediatric Emergency Care 27: 443-448.

3. Hess D (2004) Retrospective Studies and Chart Reviews. Respiratory Care 49: $1171-1174$.

4. Centers for Disease Control and Prevention (2008) Adding power to our voices: A framing guide for communicating about injury.

5. Centers for Disease Control and Prevention (2008) Data collection methods for program evaluation: Observation.

6. Müller-Riemenschneider F, Pereira G, Villanueva K, Christian H, Knuiman $M$, et al. (2013) Neighborhood walkability and cardiometabolic risk factors in australian adults: an observational study. BMC Public Health 13: 755.

7. Cerdá M, Morenoff J, Hansen B, Tessari Hicks K, Duque L, et al. (2012) Reducing violence by transforming neighborhoods: a natural experiment in Medellín, Colombia. American Journal of Epidemiology 15: 1045-1053.

8. Ferdinand A, Sen B, Rahurkar S, Engler S, Menachemi N (2012) The relationship between built environments and physical activity: a systematic review. American Journal of Public Health 102: e7-e13.

9. Roe J, Thompson C, Aspinall P, Brewer M, Duff E, et al. (2013) Green space and stress: evidence from cortisol measures in deprived urban communities. International Journal of Environmental Research and Public Health 10: 40864103

10. Han-Markey T, Wang L, Schlotterbeck S, Jackson E, Gurm R, et al. (2012) A public school district's vending machine policy and changes over a 4-year period: implementation of a national wellness policy. Public Health 126: 335337.

11. Pitts A, Burke W, Adams J (2013) Marketing messages in food and alcoho magazine advertisements, variations across type and nutritional content of promoted products: a content analysis. Journal of Public Health Jul 25.

12. Basch CH, Hammond R, Guinta A, Rajan S, Basch CE (2013) Advertising of Toothpaste in Parenting Magazines. Journal of Community Health May 15.

13. Basch CH, Ethan D, Rajan S (2013) Price, Promotion, and Availability of Nutrition Information: A Descriptive Study of a Popular Fast Food Chain in New York City. Global Journal of Health Science 5: 73-80.

14. Wansink B, Payne CR (2008) Eating behavior and obesity at Chinese buffets Obesity 16: 1957-1960

15. Halder AK, Molyneaux JW, Luby SP, Ram PK (2013) Impact of duration of 
Citation: Rajan S, Basch CH, Ethan D (2013) Observational Data Collection of Environmental and Behavioral Characteristics: Strengths, Limitations, and Implications for Health Communication. J Mass Communicat Journalism 3: e141. doi:10.4172/2165-7912.1000e141

structured observations on measurement of handwashing behavior at critical times. BMC Public Health 2: 705.

16. Flay BR, Burton D (1990) Effective mass communication strategies for health campaigns. In Atkin, C, Wallack, L (Eds) Mass communication and public health: Complexities and conflicts. Sage Publications, Inc, Thousand Oaks, CA 121: 129-146.

17. Cates JR, Ortiz R, Shafer A, Romocki LS, Coyne-Beasley T (2012) Designing messages to motivate parents to get their preteenage sons vaccinated against human papillomavirus. Perspectives on Sexual and Reproductive Health 44 $39-47$.

18. McCormick LC, Pevear J 3rd, Xie R (2013) Measuring levels of citizen public health emergency preparedness, Jefferson County, Alabama. Journal of Public Health Management and Practice 19: 266-273.

19. Bekalu MA, Eggermont S (2013) Media use and HIV/AIDS knowledge: a knowledge gap perspective. Health Promotion International May 3. 